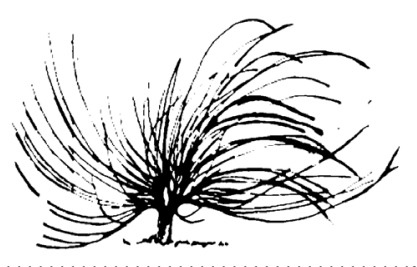

\title{
Desafíos de la docencia universitaria desde la pedagogía de la autonomía
}

\author{
Stephanie Valdivia Durán ${ }^{1}$ \\ Ministerio de Educación Pública \\ Costa Rica \\ tefa0893-e@hotmail.com
}

\begin{abstract}
Resumen
La educación secundaria costarricense se encuentra rezagada en cuanto a su calidad, pues sigue imperando el modelo educativo tradicional. A raíz de esto, el perfil de estudiante que entra a la universidad se caracteriza por no estar acostumbrado a tener criterio personal o asumir la responsabilidad plena sobre su proceso educativo, es decir no se le educa desde la autonomía. Por esta razón, la docencia universitaria se enfrenta ante el reto de fomentar procesos educativos desde la autonomía, con el fin de que el estudiantado encuentre en la universidad un espacio para desenvolverse de manera integral entre lo personal y lo profesional, desarrollando habilidades que le permitan convertirse en un ser con criterios propios. Uno de los principales retos se encuentra en la mediación pedagógica, principalmente con el estudiantado de primer ingreso, pues al no venir preparados con ciertas habilidades necesarias para la vida universitaria, es el docente universitario quien se las empieza a potenciar.
\end{abstract}

Recibido: 26 de noviembre de 2018. Aprobado: 12 de setiembre de 2019.

http://dx.doi.org/10.15359/rep.14-2.2

1 Docente de secundaria con el Ministerio de Educación Pública y en el sector privado. Integrante del Colectivo A de Libertad, de la Editorial Ediciones Libres y del Centro de Amigos para la Paz. Bachiller en la Enseñanza de los Estudios Sociales y la Educación Cívica en la Universidad Nacional. Licenciada en Pedagogía con énfasis en Didáctica en la Universidad Nacional. Actualmente, cursa la Maestría en Educación con énfasis en Pedagogía Universitaria en la Universidad Nacional. 
Es por ello que, el presente ensayo pretende desarrollar, mediante cuatro aspectos, los principales desafíos a los que se enfrenta un docente universitario para realizar una praxis pedagógica en la que enfoque su mediación desde la pedagogía de la autonomía de Paulo Freire.

Palabras clave: autonomía, desafíos, docencia universitaria, mediación, Paulo Freire, pedagogía, praxis pedagógica.

\begin{abstract}
Costa Rican high school education is stagnant in terms of quality due to a prevailing traditional educational model. Based on this, a student's profile that enrolls at the university can be defined by an individual without personal opinion and lack of responsibility for his or her educational process. In order words, this student is not educated for his or her autonomy. For this reason, university teaching faces the challenge to foster autonomy processes in the students, in order to let them find in the university the space to develop personally and professionally. One of the pedagogical challenges happens mainly with first-year students, as they do not come prepared with certain necessary skills for university life; it is the professor who strengthens those abilities. One of the main tasks is found in pedagogical mediation. For this reason, this essay aims to address four specific aspects that university teachers should face to perform a pedagogical praxis focusing their mediation on Freire's Pedagogy of Autonomy.
\end{abstract}

Keywords: Autonomy, education challenge, university teaching, mediation, Paulo Freire, pedagogy, pedagogical praxis

El educador está llamado a sembrar la semilla de la comprensión histórica en los educandos como sujetos de transformación social, económica, política y cultural. Esto solo puede ser logrado con un educador consciente de la realidad existencial de los educandos y la suya propia. 


\section{Introducción}

$\mathrm{E}$

1 presente ensayo surgió a partir de un proceso de reflexión que se llevó a cabo durante tres meses en el módulo de Praxis pedagógica en el contexto universitario, de la Maestría en Educación con énfasis en Pedagogía Universitaria de la Universidad Nacional de Costa Rica. A lo largo de dicho curso, se realizaron diversas actividades reflexivas que condujeron a la necesidad de escribir sobre los desafíos a los que se enfrenta la docencia universitaria, cuando intenta implementar la pedagogía de la autonomía.

Dicho enfoque se eligió, dentro de las experiencias vividas, hubo interacción con estudiantes de primer ingreso en el área de docencia en diferentes disciplinas, tal como inglés y música; las edades oscilaron entre los 17 y 21 años. Las preguntas realizadas a este grupo se centraron en la descripción de la experiencia educativa en el colegio y en la universidad.

Por esta razón, los estudiantes describieron a la universidad de manera comparativa con la educación que recibieron en secundaria, viendo el espacio universitario como un lugar para acercarse a la autonomía. En este sentido, se comprende que para desenvolverse en la universidad es necesario que el estudiantado aprenda a desarrollarse de manera autónoma y no tan dependiente como tiende a suceder en la educación secundaria. Por esta razón, el propósito de este ensayo es visibilizar los retos a los que se enfrenta la docencia universitaria, en cuanto a las estrategias de mediación, cuando se trata de generar procesos educativos basados en la autonomía, esto tomando como base la pedagogía de la autonomía de Paulo Freire.

De esta manera, en primera instancia se va a problematizar sobre el perfil general del estudiantado que egresa de las aulas de secundaria, con el fin de conocer cuáles son los retos que enfrenta el cuerpo docente universitario ante dichas características; asimismo, identificar las demandas que exige la universidad, al ser esta un espacio de humanismo, autonomía y criticidad. Seguidamente, se va a contextualizar sobre qué se entiende por pedagogía de la autonomía y cuál es su importancia, esto para poder entender desde qué posicionamiento se comprenden estos desafíos. Por último, como tercer aspecto, se van a desarrollar los retos, en cuanto a mediación pedagógica, a los que se enfrenta un docente universitario que pretende fomentar la autonomía en sus estudiantes. 


\section{De la educación secundaria a la educación universitaria}

Si bien es cierto, la educación en Costa Rica posee bastante cobertura, son pocos los avances que se han dado en la calidad de la educación secundaria. A pesar de los esfuerzos que se hacen en la formación de los profesionales para que practiquen la docencia con más consciencia y criticidad, existen diversos problemas estructurales que siguen generando un contexto propenso a la reproducción de la educación tradicional, tal como la exigencia de cumplir con una serie de contenidos, la evaluación homogénea, la sobrepoblación estudiantil, la exagerada carga administrativa a la que se enfrentan los docentes, entre otros.

Por esta razón, aunque haya docentes dotados de una buena formación pedagógica, el condicionamiento estructural del sistema lleva en reiteradas ocasiones a que no logren poner en práctica muchos de sus conocimientos, o que logren hacerlo, pero no en todo momento. De esta manera, en pleno 2018, el contexto educativo se encuentra deteriorado, obsoleto y con una calidad precaria, donde el estudiantado se encuentra limitado a memorizar, no se le enseña a ser crítico, su aprendizaje se reduce a aprender los contenidos que la estructura del sistema quiere que aprenda y no se le permite alzar su voz ni darse cuenta de que es un sujeto capaz de crear y transformar; es decir existe el acceso a la educación, pero no precisamente a una de calidad y mucho menos a una enseñanza desde y para la autonomía.

Con respecto a lo anterior, Solano (2017), menciona que "la educación actual no abarca las demandas que el mundo actual requiere y exige, en especial cuando las políticas de los Estados no están vinculadas a las necesidades locales" (p. 28). Con esto se ve cómo la educación secundaria, al seguirse abordando desde lo tradicional, no está promoviendo habilidades que le permitan, al estudiantado, responder a las demandas de la sociedad; así como tampoco está potenciando las competencias necesarias para la vida de las personas jóvenes, al ingresar al sistema universitario, es decir, no está brindando una educación de calidad.

En este sentido, Avilés (2012), rescata la definición de educación de calidad como la que asegura a la juventud "la adquisición de conocimientos, capacidades, destrezas y actitudes necesarias para equipararles para la vida adulta" (p. 114). Sobre esto, es necesario destacar que dichas características, entre otras, no se pretenden rescatar precisamente en miras a una "vida adulta", sino más bien para una vida autónoma; es 
decir que el tipo de estudiantado que se está egresando de la secundaria y que ingresa a la universidad, no sale con un perfil que cuenta con habilidades para desenvolverse en el contexto universitario.

De esta manera, le ha correspondido a la universidad encargarse de potenciar las capacidades del estudiantado, para que poco a poco vaya desarrollando habilidades como la criticidad, el pensamiento propio, la problematización de diversos temas y la autonomía en cuanto a su propio proceso de aprendizaje. Por ejemplo: los estudiantes de primer ingreso, con los que se entró en diálogo para realizar este escrito, manifestaron que el proceso de aprendizaje vivido en secundaria se redujo a responder exámenes memorísticos y que no hubo espacios en los que pudieran dar sus opiniones. Contrario a esto, comentaron que la universidad sí les ofreció espacios en los que pudieron dar sus opiniones y se enfrentaron a evaluaciones diversas, convirtiendo a la universidad en el sitio donde se les dio un lugar como sujetos y no como objetos.

Con esto se logra visualizar el reto que implica para la docencia universitaria, tratar de que los espacios de enseñanza-aprendizaje (entendiendo que desde una posición freireriana, no es posible que se dé el acto de enseñar sin aprender) fomenten la autonomía que el estudiantado necesita para poder aprender a crear sus propios criterios, mostrar la gama de métodos con los que se les puede evaluar y además potenciar una educación que, desde lo autónomo, les lleve a deconstruirse y volverse a construir de una manera más integral, donde haya crecimiento académico y también para la vida. Con esto resulta necesario aclarar que es aprendizaje para la vida, no para la "edad adulta".

A raíz de lo mencionado, es importante conocer qué se entiende por autonomía desde la percepción de Freire y cuál es la importancia de fomentarla en el contexto universitario. En este sentido, se comprende la autonomía como un proceso de decisiones y de humanización que se construye a lo largo de la historia y partiendo de las decisiones que se van tomando durante la vida (De Fraga, 2015). Para llegar a dicha dinámica, es necesario tener consciencia de sí mismo, ya que dicha consciencia puede llevar a lecturas de mundo críticas y de cuál sería su posición como estudiante y profesional en camino; lo cual conduce a una educación que se dimensiona desde lo político.

De esta manera, la educación no se encuentra desligada de la política; pues la educación escolarizada siempre se ha utilizado desde lo monológico (Méndez, 2009) y esto ha tenido el fin de sostener la cultura 
dominante, la cual no tiene ningún interés por fomentar una educación que enseñe a pensar. Por esta razón, la autonomía resulta fundamental, pues la universidad no debería ser un espacio para, únicamente, obtener un grado académico, sino que también, debe tener espacios en los que se eduque para pensar, crear y transformar la realidad.

Para ello, es necesario tomar decisiones propias y conscientes que se pueden lograr solo desde la libertad del ser humano con respecto a las culturas dominantes. De ahí la urgencia por crear espacios de enseñanza-aprendizaje que generen una transformación y una liberación con profesionales que, más que tecnócratas, también sean capaces de llevar luchas políticas y crear o transformar desde su disciplina.

\section{Desafíos de la mediación pedagógica para un proceso pedagógico autónomo}

Con base en la descripción anterior, se ve cómo el docente universitario se enfrenta a diversos retos pedagógicos para lograr que el estudiantado despierte su consciencia de sujeto y abandone la condición de objeto a la cual se le llevó, durante muchos años, en la educación tradicional.

Para efectos de este ensayo se va a desarrollar el reto de las estrategias de mediación que puede utilizar un docente universitario para fomentar la autonomía; tomando en cuenta cuatro aspectos principales: diversidad, carga académica, disfrute y tiempo pedagógico.

Lo primero que debe tener presente un docente es que su grupo no es homogéneo y que el alcance de la libertad para cada estudiante es diferente; es decir, el primer elemento es la diversidad. Es necesario que un docente universitario tenga presente en su praxis que no solo existe lo diferente, sino que existen las diversidades pues, según Sacristán (s. f.) "la diversidad responde a la circunstancia de los sujetos de ser distintos y diferentes" (p. 1) y se debe aprender a vivir con ella y trabajar a partir de ella. Por tal razón, un docente universitario debe pensar sus mediaciones pedagógicas contemplando las diversidades que hay en el aula y trabajar desde las fortalezas del estudiantado. Esto implica llevar al estudiantado a reconocerse a sí mismo e identificar cuáles son esas fortalezas. Este reconocimiento es un paso a la autonomía de saber por sí mismo cuál es el papel que desea cumplir en la transformación de la realidad. 
De igual forma, esto conlleva responsabilidad y compromiso por parte del docente, al crear espacios en los que pueda conocer al estudiantado y la personalidad general del grupo con el que va a trabajar. De esta manera, el docente debe procurar que sus estudiantes se sientan incluidos en el proceso de enseñanza-aprendizaje, por lo que además de ser flexible con su mediación, también debe procurar que en sus clases exista un ambiente ameno en el que el estudiantado se pueda expresar sin temor a ser discriminado.

Esto se plantea como un reto, pues es claro que, por ejemplo, no todo estudiante va a tener la habilidad de trabajar al mismo ritmo que los demás, cada uno tiene su propio tiempo y proceso; sin embargo, la universidad como institución, también condiciona el tiempo con el que cuenta un docente para abarcar los temas que se les exige bajo un currículum que no siempre es flexible.

Por esta razón, es que se menciona que lo mejor sería que el docente procure utilizar estrategias que se acoplen a las habilidades del estudiantado, siempre que cada una de ellas tenga un objetivo pedagógico; y para ello, no significa que el docente va a tener que crear una estrategia diferente para cada estudiante; sino que puede utilizar estrategias que sean flexibles. Por ejemplo, en el proceso vivido por la autora, al cursar la maestría, se puso al estudiantado a realizar una reflexión sobre la praxis docente universitaria y a partir de ella, crear una metáfora, sin embargo, la manera de presentar la metáfora fue abierta, de manera que algunos estudiantes presentaron imágenes ya hechas, otros las crearon, otros las dibujaron y otros más bien realizaron una metáfora en verso. En este ejemplo, se ve como hubo una instrucción general para todo el grupo, pero hubo flexibilidad en la manera en que se podía presentar el resultado.

Un ejercicio de este tipo hace que el estudiantado pueda llevar el mismo ritmo, pero desde sus propias habilidades. En este sentido, es necesario pensar que la educación no debería ser un proceso doloroso; sino que más bien debe ser un espacio de disfrute. Este aspecto es importante destacarlo para introducir el segundo reto que se debe tomar en cuenta en la mediación pedagógica desde la autonomía, que es la carga académica. Los estudiantes con los que se interactuó en el módulo manifestaron que en muchas ocasiones los docentes asignan tanta carga académica que terminan haciendo sus trabajos por obtener una nota. 
Sobre esto, es necesario que un docente universitario intente comprender que la emancipación no es un asunto que le compete solo al estudiantado; sino también al docente mismo y es importante reconocer que la sobrecarga de trabajos es una de las características que sigue heredando la educación tradicional. Es claro que siempre se le debe exigir al estudiantado para que en su propio proceso se convierta en un ser para sí, pero en el momento en el que el proceso educativo se sobrecarga de asignaciones académicas, el estudiantado deja de reflexionar y pierde el norte de lo que puede aprender en ese proceso, centrándose más bien en trabajar por obtener una nota suficiente para aprobar un curso que le llevará a la obtención de su grado académico que al final es lo que exige el mercado.

Dicha dinámica termina contextualizándose en una educación bancaria y no en una liberadora. Es importante recordar que, para desarrollar una pedagogía de la autonomía, es necesario vincularse con una educación que lleve a la libertad y no al simple depósito de contenidos, pues en la bancaria no se crean las posibilidades de producir ni construir conocimiento, sino que se limita a transferirlo, aspecto que sucede mucho cuando hay exceso de asignaciones. Esto se convierte en una contradicción para un docente que promueva un discurso desde lo autónomo, pues tal como plantea Freire (2004), en el libro Pedagogía de la autonomía, como docente no se puede estar pronunciando bellas frases epistemológicas y políticas sobre la teoría, si no se está enseñando esa teoría mediante el ejemplo, pues si no se es consecuente "me vuelvo tan falso como quien pretende estimular el clima democrático en la escuela por medios y caminos autoritarios" (p. 16).

Aunado a esto, se encuentra el tema de las lecturas como parte de la mediación pedagógica universitaria. Si bien es cierto, las lecturas son fundamentales para un proceso de aprendizaje desde la autonomía, en la universidad hay una tendencia a sobrecargar al estudiantado de textos, sin comprender que en un ciclo el estudiante lleva más de un curso y en cada uno de ellos hay cargas similares. El resultado de esto es que el estudiantado se dedique a hacer lecturas rápidas, pues entre las asignaciones, las lecturas y la vida personal, no queda tiempo suficiente para realizarlas con el debido análisis. Sobre esto Freire y Shor (2014), manifiestan que un libro será provechoso en la medida que haya motivación al realizar el propio acto de la lectura; es decir que un libro se 
debe manejar con seriedad, pues leerlo es establecer una conversación con él.

Esto no quiere decir que no se deba leer. Sí es necesario hacerlo, pero también es fundamental que al leer haya seriedad y compromiso y esto no se puede lograr si las asignaciones de lecturas y tareas se hacen en exceso. Asimismo, es preciso decir que un docente universitario no debe reducir la lectura a lo meramente textual, pues hay otras maneras de realizar lectura y una de ellas es la realidad. En este sentido, Freire y Shor (2014), plantean que existen otros espacios, además de la escuela, que se pueden utilizar para leer y se refieren al de los movimientos sociales. Esto implica que, si se va a ver en clase un tema sobre ecología, es importante leer sobre ello; pero también, es importante hacer una lectura que no es textual y que más bien se da llevando al estudiantado al interior de un movimiento ecologista, pues es dentro de estos movimientos donde muchas veces se aprenden aspectos, de una educación autónoma y liberadora, que no podríamos ver en un texto.

Por otro lado, en cuanto a las asignaciones, no se pretende decir que no se deban realizar, sin embargo, una manera de hacerlo es lo abordado con respecto a la diversidad, lo cual implica realizar indicaciones flexibles que permitan que el estudiantado disfrute de lo que se solicita en el proceso de enseñanza-aprendizaje, de manera que si el estudiante lo que más disfruta en su tiempo libre es hacer música, entonces pueda producir conocimiento y aterrizar esa construcción a la realidad mediante la música. $\mathrm{O}$ en cuanto a los textos, se podría ser flexible al dejar que en algunas ocasiones sea el estudiantado quien escoja un texto que quisiera realizar con respecto a un tema asignado por el docente, pues así se estaría vinculando al estudiante en la construcción de su propio proceso educativo.

Si se pone en práctica lo anterior, la educación universitaria podría ser disfrutada y no precisamente sufrida; y es con esta idea con la que se introduce el tercer reto al que se enfrenta un docente universitario en la mediación pedagógica autónoma: el disfrute. Es claro que las universidades preparan al estudiantado para su vida profesional; pero desde una perspectiva crítica y liberadora, también los debe guiar para ser sujetos de transformación en la sociedad. Es con esta idea donde se ve que, desde la pedagogía de la autonomía, el acto educativo tiene dimensión política, pues este no debe girar en torno al mercado, sino a las exigencias necesarias para la construcción de un mundo mejor. 
Esto se aborda, desde la pedagogía de la autonomía, como la dicotomía entre la ética del mercado versus la ética de la educación. En este sentido, desde la perspectiva freireriana, se plantea que, si se busca un mundo para las personas, es necesario hacer frente a la ética del mercado, la cual Freire relaciona con la globalización que, mediante un discurso oculto, busca encubrir la intensión de incrementar la riqueza de unos pocos y verticalizar la pobreza y la miseria de millones (Freire, 2004).

En este sentido, la universidad, al estar institucionalizada, responde de alguna manera al mercado; sin embargo, es necesario recordar constantemente que la labor del docente es su compromiso con su ética educativa desde su posicionamiento político y ello implica conservar la rebeldía, a la que tanto invita Freire, en favor de una educación para la liberación; máxime si el posicionamiento de dicha docencia es desde la autonomía; y para ello debe crear estrategias que no reproduzcan las dinámicas que impone el sistema capitalista. Por ejemplo, potenciar el trabajo tanto individual como colectivo, estimular la creatividad la cual no implica caer únicamente en lo lúdico, fomentar la criticidad, conducir siempre a que el estudiantado busque su propia libertad.

Así, en cuanto el estudiantado logre comprender que su proceso educativo es más que un título de grado para insertarse en el mercado laboral, se dará cuenta que puede disfrutar de su camino a la libertad. De igual forma, cuando el docente universitario comprenda que la universidad va más allá de lo academicista, logrará ver que no existe algo malo en que la risa, o cualquier expresión humana que quiera ser aniquilada por el sistema capitalista, sea parte del acto educativo.

Ahora bien, existen dos aspectos que limitan la mediación pedagógica autónoma en las universidades. El primero es el tiempo y el segundo es la disposición que tenga el estudiantado en su proceso educativo; el segundo será desarrollado en otro apartado. En cuanto al tiempo, se plantea como un reto para los docentes universitarios, pues este es precisamente, uno de los problemas estructurales que muchas veces limitan los procesos de aprendizaje.

La universidad como estructura institucional debe tener planes curriculares que la docencia ha de cumplir; a raíz de esto muchas veces los docentes se ven obligados a acelerar los procesos educativos con tal de terminar de desarrollar todos los contenidos que se deben ver en un curso. Esto en ocasiones limita el tipo de mediación que se va a utilizar, 
pues deben ser pensadas con miras a que abarquen los aspectos mencionados (diversidad, carga académica y disfrute) y además que el tiempo permita desarrollarlas.

Esta problemática se agrava cuando el tiempo de las universidades responde a las competencias del mercado; esto se refiere a que el mercado laboral exige profesionales con títulos de grado, sin importar mucho, si se encuentran bien capacitados para ejercer la profesión. A raíz de esto, ha aumentado la apertura de universidades que ofrecen títulos de grado en poco tiempo, aunque esto implique una preparación más precaria.

Debido a esto, las universidades comienzan a ofrecer programas de estudio más cortos que, si bien es cierto, permiten que la universidad siga inmersa en la dinámica del sistema, limita el tiempo pedagógico de los docentes el cual, según Sanjurjo (s. f.), se va a entender como "el tiempo en que organizadamente interactúan docente-alumno-conocimiento" (p. 2).

Con esto se puede ver cómo el tiempo puede condicionar el acto educativo, pues el proceso de cada estudiante no lleva un mismo ritmo, lo cual provoca que, en algunas ocasiones, se den procesos educativos homogeneizantes en los que se abordan los contenidos y las evaluaciones al estudiantado bajo un mismo ritmo y a un mismo nivel, sin tomar en cuenta la diversidad dentro del aula. Esto, aunque sea un problema estructural del sistema, no deja de representar un reto para un docente que intente realizar una mediación desde la autonomía y que a la vez atienda las exigencias del estudiantado y de la institución.

\section{De la rebeldía y la desesperanza}

Tal como se mencionó, además del tiempo, un reto al que también se enfrenta la docencia universitaria, es la disposición que tenga el estudiantado ante su proceso de aprendizaje, pues muchas veces estos se resisten a las prácticas pedagógicas del docente. En este sentido, hay que ser rigurosos al identificar la razón por la cual un estudiante se puede resistir al proceso educativo planteado por el docente. Una razón podría ser la rebeldía ante prácticas tradicionales obsoletas; mientras que otra puede responder a un sentimiento de desesperanza en el que el estudiantado no se sienta capaz de aprender a ser autónomo y libre.

Con respecto a la primera razón, los docentes, quienes han pasado años de formación bajo una educación tradicional, encuentran difícil 
desprenderse de los males que les ha dejado ese proceso educativo; a raíz de esto, sin darse cuenta en algunas ocasiones, repiten ciertas prácticas que generan rechazo por parte de un estudiantado que comienza a buscar un mundo diferente, pues tampoco se puede partir de que el educando está completamente alienado. Sobre esto, la visión freireriana sostiene que el educando debe mantener vivo su gusto por la rebeldía, pues esta agudiza su curiosidad y estimula su capacidad de arriesgarse; logrando así ser inmune ante las fatalidades del "bancarismo" (Freire, 2004). Para evitar esto es necesario que la mediación pedagógica direccione al estudiantado a asumirse como ser pensante, comunicante, transformador, creador, realizador de sueños, capaz de sentir rabia, porque es capaz de amar.

Por otro lado, la segunda razón apela a la resistencia por desesperanza. Esta se da cuando un docente promueve prácticas que sacan de la zona de confort al estudiantado y a las cuales este se resiste a asimilarlas por simple desesperanza. Como ya se ha mencionado, la educación, al tener una dimensión política, no está exenta de las fatalidades que provoca el capitalismo, de allí que el estudiantado se vea desesperanzado o fatalista ante la propuesta de aprender a ser autónomo y asumir la responsabilidad que acompaña la libertad. Sobre esto Freire (2004), afirma que la desesperanza se puede concebir solo como un momento de transición, pero no como una posición permanente; pues para él, permanecer en esta condición, sería una traición a nuestra propia naturaleza esperanzadora y buscadora.

A pesar de ello, sí hay estudiantes que se empeñan en no querer abandonar su condición de comodidad y esto implica reforzar la exigencia en las mediaciones pedagógicas, pues según Freire, es necesario convencer a los jóvenes de que la realidad puede ser transformada, que la historia no termina con la historia individual de cada persona, por lo que es necesario demostrarles que "la historia no es un conjunto de acontecimientos que suceden mecánicamente, sino el producto de la decisión de los hombres" (Masi, 2008, p. 80). Esto implica asignar responsabilidad y compromiso no solo del docente, sino del estudiantado.

Ahora bien, detectar el tipo de resistencia en la que se encuentra el estudiantado es un desafío más, pues esto implica que el docente universitario se encuentre en una constante reflexión de su praxis pedagógica; tanto desde el plano individual como desde lo colectivo. 
Asimismo, esto exige que el docente constantemente observe y realice una lectura del estudiantado.

La reflexión individual implica que el docente sea capaz de reconocerse como un ser histórico e inacabado que siempre puede mejorar, en este sentido Solano (2013), sostiene que la persona al reconocerse como ser histórico, abre paso a "reconocer sus errores pasados, para actuar en el presente con miras hacia un futuro más prometedor" (párr. 10). Esto, desde la pedagogía de la autonomía se considera un elemento muy importante, pues se parte de que ninguna persona es acabada; nadie sabe todo, siempre se aprende, se conoce, se crea, se transforma. Y para la docencia universitaria esta reflexión es necesaria para mejorar su propia praxis.

Por otro lado, dicha reflexión también se debe hacer desde lo colectivo, tomando en cuenta la voz del estudiantado. Como ya se ha mencionado, la docencia requiere investigación constante, tomando en cuenta la crítica y la reflexión sobre la praxis; no obstante con esto es necesario recordar que dentro de esta dinámica de reflexión el estudiantado toma un papel importante, pues tal como dice Freire en el libro de Pedagogía de la Autonomía, no hay docencia sin discencia; lo cual significa que en el proceso de aprendizaje ninguno de los sujetos toma la posición de objeto, pues "quien enseña aprende al enseñar y quien aprende enseña al aprender" (Freire, 2004, p. 8).

En este sentido, el hecho de que un docente universitario le brinde un espacio al estudiantado para que comunique sugerencias de cómo podría mejorar su praxis, muestra mediante el ejemplo cómo se aprende desde la autonomía, asumiendo el reto de cambiar, el inacabamiento, la dialogicidad y sobre todo le muestra al estudiante que su opinión más que importante es necesaria para lograr la transformación, este debiera ser el caso de la praxis docente universitaria.

\section{Conclusiones}

El propósito de este ensayo fue problematizar algunos de los retos con los que se enfrenta la docencia universitaria, para fomentar la autonomía en estudiantes con el perfil que poseen al egresar de la educación secundaria, todo desde la mediación. En este sentido, se plasmó que el sistema educativo de secundaria no está educando a los jóvenes ni para, ni desde la autonomía, lo cual ha significado un reto para la universidad y para la docencia universitaria, pues son estos quienes desde 
su praxis pedagógica intentan guiar al estudiantado para que comprenda que, contrario a lo que les dice la educación tradicional, su opinión sí tiene validez, son capaces de ser críticos y son sujetos capaces de asumirse como seres pensantes, transformadores, creadores y realizadores de sus propios sueños.

Los retos a los que se enfrenta el docente universitario responden a implementar mediaciones pedagógicas que tomen en cuenta la diversidad, un adecuado planteamiento de las cargas académicas que se le va a asignar al estudiantado, no dejar de lado el disfrute en su praxis sin caer en lo meramente lúdico, todo esto tomando en cuenta las limitaciones, como lo son las condiciones estructurales del sistema, tal como el tiempo, el currículum y la desesperanza que el neoliberalismo siembra en el estudiantado.

Ante esto es necesario que la docencia universitaria tenga claridad en su posicionamiento político y sea fiel a este, de manera que no permita dejarse llevar únicamente por las limitaciones y trate de manejar la mayor coherencia posible entre su discurso y la praxis pedagógica. Asimismo, que tengan la disciplina de reflexionar constantemente su praxis pedagógica desde lo individual como desde lo colectivo, es decir en conjunto con el estudiantado.

Esto último es algo que la docencia no puede olvidar en su quehacer, pues la labor del educador es importante en la medida que, tal como lo dice la frase al inicio del ensayo, es quien está llamado a sembrar la comprensión histórica en los educandos como sujetos de transformación social, económica, política y cultural; y la universidad más que formar profesionales debe procurar egresar seres humanos con consciencia de agentes de transformación, pues al final el mundo no se cambia en las aulas, sino en las calles.

\section{Referencias}

Avilés, G. (2012). Retos y desafíos de la educación costarricense. InterSedes: Revista de las Sedes Regionales, 13(26). Recuperado de http://www.redalyc.org/pdf/666/66624662006.pdf

De Fraga, R. (2015). Diccionario de Paulo Freire. Lima: Consejo de Educación Popular de América Latina y el Caribe.

Freire, P. y Shor, I. (2014). Miedo y osadía. La cotidianidad del docente que se arriesga a practicar una pedagogía transformadora. Buenos Aires: Siglo Veintiuno. 
Freire, P. (2004). El grito manso. Buenos Aires: Siglo veintiuno.

Freire, P. (2004). Pedagogía de la Autonomía. Sao Paulo: Paz e Terra S. A. Recuperado de http://cidac.filo.uba.ar/sites/cidac.filo.uba. ar/files/revistas/adjuntos/UNIDAD\%207\%20-\%20Freire $\% 20$ Paulo\%20-\%20Pedagog\%C3\%ADa\%20de\%201a\%20autonom $\% \mathrm{C} 3 \%$ ADa.pdf

Masi, A. (2008). El concepto de praxis en Paulo Freire. En Gadotti, M. et al. (Comp.), Paulo Freire. Contribuciones para la pedagogía (pp. 75-82). Buenos Aires, Argentina: CLACSO. Recuperado de http://bibliotecavirtual.clacso.org.ar/ar/libros/campus/freire/ 09Masi.pdf

Méndez, J. (2009). Educación Intercultural y Justicia Cultural. Recuperado de https://espanol.free-ebooks.net/ebook/ Educacion-Intercultural-y-Justicia-Cultural

Sacristán, J. (s. f.). La construcción del discurso acerca de la diversidad y sus prácticas. Aulas de Innovación Educativa, 81. Recuperado de http://altascapacidadescse.org/pdf/la_construccion_del_discurso.pdf

Sanjurjo, L. (s. f.). Volver a pensar la clase. Presentación al $2{ }^{\circ}$ Congreso Nacional de Educación del Este Cordobés "Nuevas perspectivas didácticas en el aula". Recuperado de https://des-for.infd. edu.ar/sitio/upload/1466485945.Sanjurjo_Liliana_Volver_a_ pensar_la_clase.pdf

Solano, J. (2013). El ser histórico en la pedagogía crítica. Costa Rica: EquipoCrítica.org. Recuperado de https://www. equipocritica.org/reflexion-editorial/editoriales-anteriores/ el-ser-historico-en-la-pedagogia-critica/

Solano, J. (2017). Una mirada crítica de las dictaduras latinoamericanas durante la Guerra Fría mediante la Nueva Canción Latinoamericana (tesis de grado). Universidad de Costa Rica, Costa Rica. 
